\title{
Research on CBTC Function of Train Control System
}

\author{
Shi Jiateng \\ Beijing Jiaotong University Haibin College, Hebei, Cangzhou 061100,China
}

Keywords: CBTC, vehicle onboard system, cause-effect diagram, Test case

\begin{abstract}
Recent years, with increasing urban population, urban traffic's pressure is increasing. Urban mass transition is in the period of rapid development. The new generation of train control system-CBTC (Communication Based Train Control) is applied to the constructions of urban mass transition. In CBTC system, vehicle onboard system plays an important role about security and effectiveness of train. The function of vehicle onboard system is directly connected with the traffic safety. Therefore, before the vehicle equipment system puts into operation, we must complete rigorous tests and verify the functions. In the test, the test cases directly affect the quality of test works. So this paper is focused on test methods and specific test case for vehicle onboard system. The functions of vehicle onboard system should be modular. Modularization of the functions could reduce the number of test cases and improve the efficiency of the test.
\end{abstract}

\section{Introduction}

With the rapid development of China's economy, the large and medium-sized cities are constantly expanding, and the urban covering area and urban population are both steadily on the increase again. What's more, the nation's urban rail transit construction has entered a rapid development period. Urban rail transit belongs to the green traffic system. It has many characteristics, such as great load carrying capability, energy conservation, punctuality, and the advantage without affecting the operation of ground transportation, etc. In this way it can largely alleviate the road traffic pressure of various large and medium-sized cities. According to the incomplete statistics of relevant departments, at present the metro daily passenger flow volume of the big cities like Beijing and Shanghai has already broke through 6 million, and judging from the present condition, the figure can only increase. In order to ensure that the urban rail transit can run more safely and effectively, the rail transit construction of each city gives up to choose the use of the original the Track Circuit Based Train Control System (TBTC) and adopts The new generation of train control system-CBTC (Communication Based Train Control) .

CBTC system has realized the moving block, so it makes the train not be restricted by the situation, which the train runs on the same block section in the original TBTC system. CBTC system can greatly improve the traffic density of train so as to meet the modern urban rail transit's operational requirements of high speed, high density and high capacity ${ }^{[1]}$. 1.2.4CBTC system structure

In 2004, IEEE RTVICS (Rail Transit Vehicle interface Standards Committee) has formulated and promulgated CBTC technical standard IEEE STD 1474.1-2004 ( Revision of IEEE STD1471.1-1999). This standard stipulates the system functional requirement of CBTC in details, such as the train intervals, the indicators of system safety performance, the functional requirements of various subsystems, etc. Using Fangshan Line subway of alstom Urbalis TM system we can design it according to this system. The system is mainly composed of six important parts, such as ATS(Automatic Train Supervision),, (Computer interlocking), ZC(Zone Controller), CC(Carborne 
Controller), DSU (Date Storage Unit) and DCS (Date Communication System). The overall structure of the system is shown in figure 1.

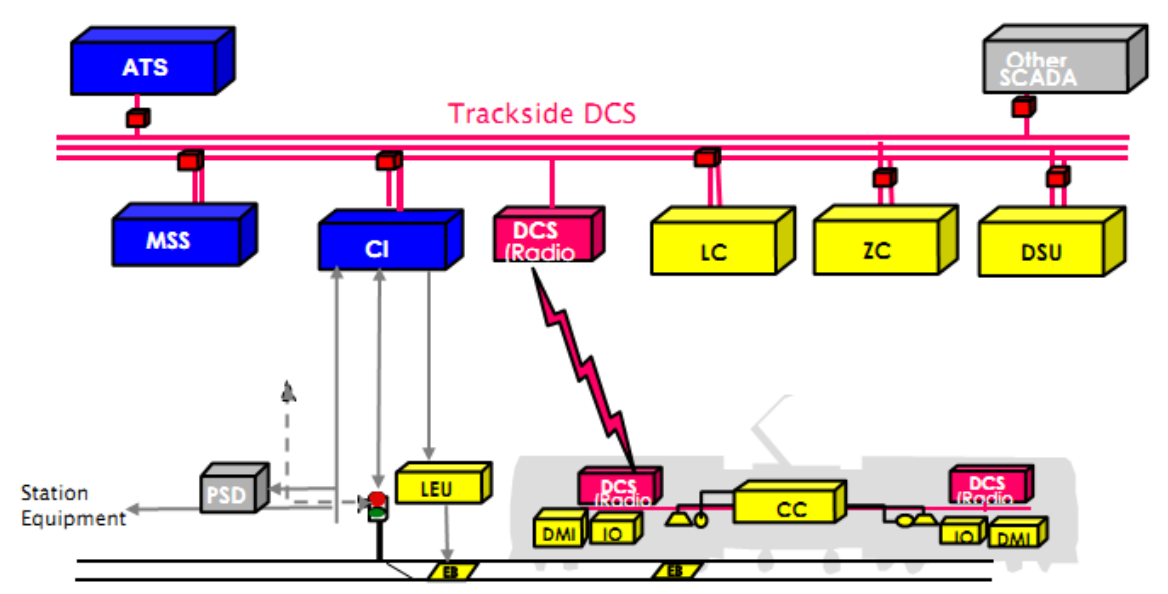

Figure 1 The basic structure of Urbalis ${ }^{\mathrm{TM}}$ system

\section{The Overall Functional Requirements Analysis of CBTC System Vehicle Onboard System}

Each Subsystem Information Transmission in CBTC System. Before we analyze the functional requirements of vehicle onboard, we need to define the interface between each subsystem under CBTC system. When CBTC system is operated, each subsystem needs to provide the information needed by other subsystems.

The exchanged information for each subsystem is shown as follows

$\mathrm{CI} \Leftrightarrow \mathrm{CC}$

CI needs to transfer the condition of turnout, signal lamp and safe door to CC;CC couples back received signal and the order to control safe door to $\mathrm{CI}$.

$\mathrm{CC} \Leftrightarrow \mathrm{ZC}$

$\mathrm{CC}$ needs to report and transfer position of train to ZC;ZC needs to couple back movement authority of variable and invariant to CC.

ATS $\Leftrightarrow$ CI

ATS transfers the order to control equipment and admission passage to CI;CI needs to couple back in time the condition of equipment to ATS.

$\mathrm{ZC} \Leftrightarrow$ ATS

ATS can transfer temporary speed limit order to ZC;ZC needs to make feedback for the condition and warning of temporary speed limit order.

$\mathrm{ZC} \Leftrightarrow \mathrm{CI}$

CI needs to transfer the condition of signal equipment and admission passage to ZC;ZC needs to couple back ATP' s block state to CI.

Vehicle Onboard Equipment's Control Function. According to the interface analysis of the data of CBTC system's all subsystems, we can find that vehicle onboard equipment is at the core position in system's information exchange and order exchange. Vehicle onboard equipment can exchange information with other subsystems by reliable wireless communication network, in addition to communicating with CI、ATS and ZC, vehicle onboard equipment also needs to make 
data transmission with train's DMI(driver management interface) to realize vehicle onboard equipment's communication function.

The train vehicle onboard equipment's control function is shown as follows:

(1) Train calculates train's current speed by train's coded odometer, checks by ground signal and makes self-localization.

(2) Train calculates running speed's curve through received M A inform ation sent by ZC to supply train's maximum running speed and object distance, in this way, to realize the safe protection for train's operation.

(3) W hen stride over the controlborder of a set of ZC and realize ZC' s autom atic sw itch, send request order to new $\mathrm{ZC}$.

(4) A ccording to train's running state at one m om ent, form ulate train 's running model and drive model at that moment.

(5) F in ish autom atic tum-back function when receiving ATS's turn-back order.

(6) C heck train w hen train is in zero-speed state to judge if train stops totally.

(7) Check the state of train door and control train door's switch; conduct safety manage to platform safe door.

\section{The Test Case Design for Testing CBTC System's Vehicle Onboard Fundamental Function and Accomplishing in Project}

Introduction to Test Software. We need help from test software to conduct the test. We use dedicated vehicle onboard test software-OMAP software in CBTC system test. This software analyzes control information of vehicle onboard equipment by loading corresponding library data, through the data from analysis, test personnel can observe in real time carborne controller's inner order information, and analyze the data in logical structure to complete vehicle onboard equipment's test. For a simple example, the software can couple back the information of train's condition, as shown as Table 1 that followed.

Table 1 data and information of train's condition

\begin{tabular}{l|l|c}
\hline Data Name & Data Type & Annotation \\
\hline TFE id & Integer & Train head's ID \\
\hline TFE block & Integer & $\begin{array}{c}\text { Block section of train } \\
\text { head's ID }\end{array}$ \\
\hline TRE block & Integer & $\begin{array}{c}\text { Block section of train } \\
\text { tail's ID }\end{array}$ \\
\hline TFE abscissa & Double & $\begin{array}{c}\text { The position of block } \\
\text { section of train head }\end{array}$ \\
\hline TRE abscissa & Double & $\begin{array}{c}\text { The position of block } \\
\text { section of train tail }\end{array}$ \\
\hline
\end{tabular}

The Table that followed is test software's sketch Fig.2 


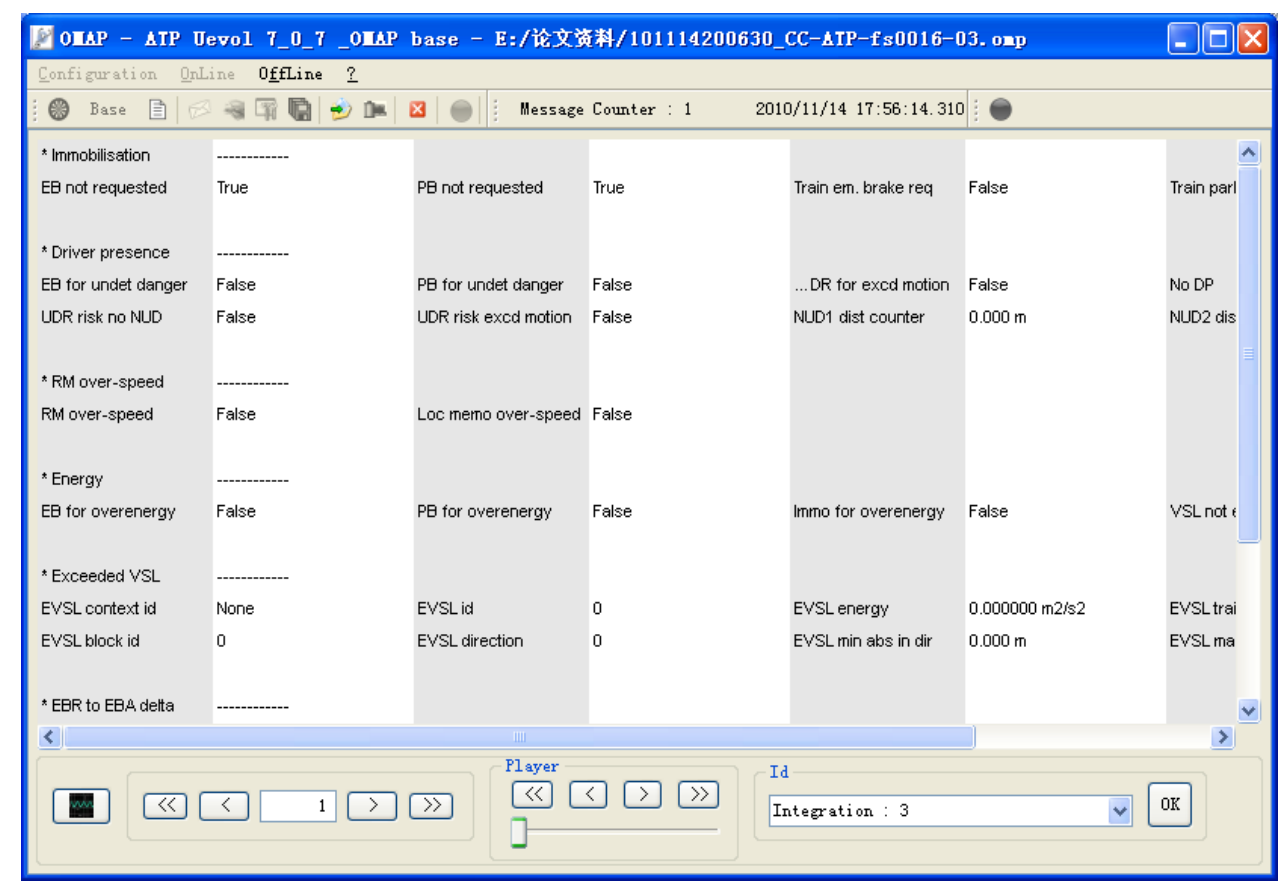

Fig.2 Sketch map of test software

The Test Case Design to Test Safe Door and Train Door. Train door and safe door are fundamental equipment to safeguard passenger's security.Because of the skyrocketing of present urban population, subway's carrying capacity will be multiplied once during rush-hours. No matter who are waiting at platform for train or who are on a train, the passenger is very large, so that either platform or train will be crowded sometime. Therefore, train door and safe door are necessary equipment to safeguard passenger's security. As a result, the test to train door and safe door would be considered carefully.

1. Introduction to Signal System and Safe door's Interface. The control of safe door(PSD)'s turn-on and turn-off is carried out by vehicle onboard(CC), in other words, carborne controller is the safety of responsible for safe door's turn-on and turn-off. Because there is no direct interface between carborne controller(CC) and safe door(PSD) so that the interface between both of they is accomplished by computer interlocking(CC) subsystem, computer interlocking(CC) subsystem as a bridge between vehicle onboard(CC) and safe door(PSD), it exports vehicle onboard(CC)'s order of turn-on and turn-off to safe door(PSD), at the same time, it sends safe door(PSD)'s state to vehicle onboard(CC). The interface between vehicle onboard(CC)、 computer interlocking(CI) and safe door(PSD) .

As shown as the sketch map above, the control of safe door's turn-on and turn-off is vehicle onboard equipment's control to safe door, computer interlocking just transfers transparently the information between vehicle onboard and safe door, it does not conduct any logical processing, and it faithfully expresses vehicle onboard' s control intention.

In normal running model, PSD system will execute operation by the output by signal system, as shown as Table 2: 
Table $2 \quad$ I/O signal system output control PSD movement

\begin{tabular}{ccc}
\hline $\begin{array}{c}\text { PSD open } \\
\text { door-safe output }\end{array}$ & $\begin{array}{c}\text { PSD close door } \\
\text {-safe output }\end{array}$ & PSD movement \\
\hline 0 & 0 & Does not move \\
1 & 0 & PSD opened door \\
0 & 1 & Closed all PSD \\
1 & 1 & Did not move( it will warn) \\
\hline
\end{tabular}

2. Time Introduction When Signal System Opens or Closes Safe Door. The wireless communication between vehicle onboard and computer interlocking will be established when train goes into PSD area, and vehicle onboard sends an order of close door by computer interlocking. After train stops totally, vehicle onboard sends an order of open door by computer interlocking(joint control with train door), this order will be keeping during train stops at station. Before train departs, vehicle onboard sends an order of close door by computer interlocking, after safe door closes and locks tight, computer interlocking will send safe door's state to vehicle onboard so that train can depart from station. After train departs from station area, because vehicle onboard loses the wireless communication with the station computer interlocking as train's departure, the order of close safe door will be renewed. The sequence chart is shown as chart 3:

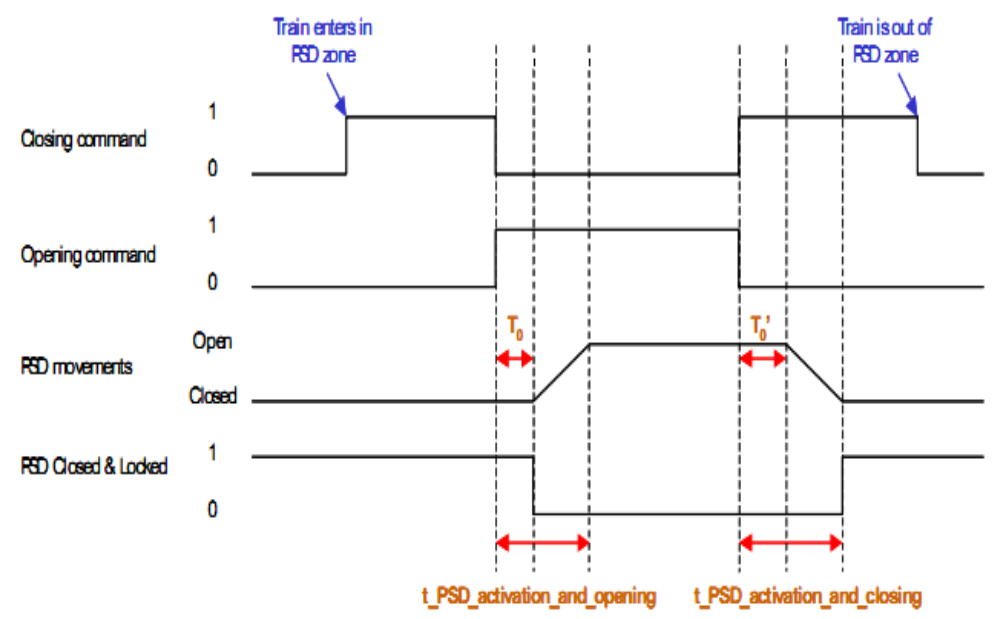

Fig. 3 The sequence chart to safe door's turn-on and turn-off

3. Test Design to Train Door and Safe Door and Analysis to Test Result. Because there is comovement relation between train door and safe door, therefore, when we design the test, we can test train door and safe door in the mean time. In this way, we can guarantee test integrity as well as improve test efficiency. As shown as the sequence chart and the interface sketch map of safe door and vehicle onboard equipment, we can get a logical flow of safe door switch.

According to the logical flow above, we need to observe and analyze the changes of parameters that followed. Parameters's definitions are as shown as Table 3. 
Table 3 Safe door and parameters of train door test

\begin{tabular}{ccc}
\hline Data Name & Data Type & Annotation \\
\hline SA PSD status & Boolean type & State of safe door in side A \\
SA TD state & Boolean type & State of train door in side A \\
SA PSD cmd & Boolean type & Control information of safe door in \\
sA TD open cmd & Boolean type & Control information of train door in \\
& & side A \\
\hline SA TD cmd & Boolean type & Control information of train door in \\
& & side A \\
SA TDCL & Boolean type & Close and lock information of train \\
& & door in side A \\
Align Status A & Aligning state of train door and safe \\
& door in side A
\end{tabular}

When train goes into station, test personnel judge whether vehicle onboard equipment can conduct the function normally through observe vehicle onboard inner processed information to the function.

\section{Summary}

Nowadays, our country's urban rail transit is in high-speed development period, CBTC system is applied by more and more rail transit company in practical project construction. In this care, we shortly need a set of complete and effective test case to test equipment to guarantee that equipment and system can operate normally. Vehicle onboard equipment plays a crucial role in CBTC system, it is key to guarantee whether train can run safely and effectively. This paper picks vehicle onboard equipment system as object of study, analyzes its functional demand and modularizes its function, on this basis, this paper uses cause-effect diagram to study and design its test case, and analyzes and studies practical test case in construction.

\section{References}

[1] Gao Chunhai. Rail Transit Train Control System Based on Communication[J]. Contemporary Urban Rail Transit.7-10.Volume 2.2007

[2] S.Morar Evolution of communication based train control worldwide.IET Seminar Digest 2010,281-288

[3] Tang Fuxing. Introduction to Urban Rail Transit System[M] Beigjing: Water Conservancy and Electricity Press

[4] Wu Haifeng. Mobile Block Train Control System Based on Communication[J].Railway Communication Signal 60-61. Volume 43.2007

[5] Zhangchao,Dong Dechun. Train Control System Based on Wireless Communication[J].Study to Urban Rail Transit.2005 
[6 Rail Transit Vehicle Interface Standards Committee of the IEEE Vehicular Technology Society IEEE Std 1474TM-1.2004. IEEE Standard for Communications Based Train Control(CBTC) Performance and Functional Requirements.2004

[7] Mooney.Kenneth A, Ghaly,Nabil N Communication based train control at MTA New York City Transit.Proceedings of the IEEE/ASME Joint Railroad Conference.1998.177-188. 